\title{
Flow Visualization Based on A Derived Rotation Field
}

\author{
Lei Zhang, Guoning Chen; University of Houston; Houston, Texas \\ Robert S. Laramee; Swansea University, UK \\ David Thompson, Adrian Sescu; Mississippi State University; Mississippi State, Mississippi
}

\begin{abstract}
In this paper, we apply a derived scalar field, called the $\Phi$ field, to assist the visualization of various flow data. The value of the $\Phi$ field at a spatio-temporal location is determined by the accumulated angle changes of the tangent directions along the integral curve starting from this location. Important properties of the $\Phi$ field and its gradient magnitude $|\nabla \Phi|$ field are studied. In particular, we show that the patterns in the derived $\Phi$ field are generally aligned with the flow direction based on an inequality property. In addition, we compare the $\Phi$ field with some other attribute fields and discuss its relation with a number of flow features, such as topology, LCS and cusp-like seeding structures. Furthermore, we introduce a unified framework for the computation of the $\Phi$ field and its gradient field, and employ them to a number of flow visualization and exploration tasks, including integral curve filtering, seeds generation and flow domain segmentation. We show that these tasks can be conducted more efficiently based on the information encoded in the $\Phi$ field and its gradient.
\end{abstract}

\section{Introduction}

Vector field analysis is a ubiquitous tool employed to study a wide range of dynamical systems. Applications involving vector fields include automobile and aircraft engineering, climate study, combustion dynamics, earthquake engineering, and medicine, among others. With the continuous increase in size and complexity of the generated data sets, there is a strong need to develop an effective abstract (or reduced) representation that addresses the complexity of data interpretation and user interaction. There is a large body of work on generating a reduced representation of the flow by classifying integral curves based on their individual attributes. These methods typically first classify the integral curves into different clusters based on their similarity [12] and then compute the representative curves for each cluster [31]. Recently, streamline and pathline attribute-based flow exploration $[6,15,20,21,23]$ was introduced. However, since the results of these techniques are represented via a sparse set of integral curves, there is no guarantee that important flow features will be captured. In addition, in order to reveal different flow behaviors, e.g., separation or vortices, different metrics are generally needed [23]. There is a need for a representation that can encode important flow information in a simple form which can be used to aid the subsequent data exploration tasks.

To address this need, Zhang et al. [32] introduce a number of attribute fields that encode global behaviors of the individual integral curves measured by certain geometric and physical properties. The scalar attribute value at each spatio-temporal position is derived via the accumulation of a given set of local flow properties along the integral curve that passes through it, which leads to an Eulerian representation of the Lagrangian behaviors of the flow particles. Note that each integral curve indicates the trajectory of a flow particle seeded at a coincident spatial position. Therefore, the computation of the derived attribute fields summarizes the behavior of the particles along their individual trajectories. The work of [32] further shows that certain discontinuities in the derived attribute fields can be observed and may be correlated with flow features, such as fixed points and other topological features, flow separation, boundary switch points, and vortices. However, it does not provide an in-depth description of the properties of the derived attribute fields other than the discontinuities. It also does not detail the applications of the derived attribute fields for flow visualization. Further the discussion focuses only on 2D flows. To address these shortcomings, we specifically focus on an attribute field generated by integrating the curvature of the flow direction (i.e., flow rotation) along integral curves, referred to as the $\Phi$ field. We also provide a more in-depth discussion of its properties and, based on these properties, we propose a number of applications based on the $\Phi$ field and apply them to the visualization of both $2 \mathrm{D}$ and $3 \mathrm{D}$ flows.

We investigate the $\Phi$ field because the encoded rotational information is intrinsically related to the curvature of the integral curve, which has been shown to be effective for characterizing integral curve behavior and revealing important flow features [12, $14,21,23]$. More importantly, the rotation is highly related to the acceleration in the unsteady case, which is the source of important flow dynamics, including separation and swirling. In summary, the contributions of this work include:

- We conduct an in-depth investigation of the $\Phi$ field and $|\nabla \Phi|$ field. The relation of the $\Phi$ field with other attribute fields is studied and a number of important properties of the $\Phi$ field are discussed. We discuss not only the discontinuities but also the inequality property of the $\Phi$ field, which can be utilized for flow visualization tasks.

- We present a $\Phi$ field based flow exploration framework unifying the $\Phi$ and $|\nabla \Phi|$ field computation. We emphasize that the framework is not explicitly designed to detect features, but represents a new and simple way of encoding flow information (i.e., via a derived scalar field) so that the subsequent flow visualization tasks, including flow segmentation, integral curve filtering and integral curve/surface seeding, can be conducted more efficiently. We apply this framework to the visualization and exploration of a number of synthetic and real-world flow data sets.

The rest of the paper is structured as follows. Section 2 reviews the previous work related to the proposed method. Section 3 briefly reviews the important concepts of vector fields and introduces the $\Phi$ field as well as its important properties. Section 4 
describes the computation of the $\Phi$ field and its gradient, and lists a number of visualization and data exploration tasks that can be assisted by the $\Phi$ field and its gradient. The applications of the $\Phi$ field-based visualization to a number of steady and unsteady flows are reported in Sections 5. Section 6 summarizes this work and discusses a number of limitations of the proposed framework.

\section{Related Work}

There is a large body of literature on the analysis and visualization of flow data. Interested readers are encouraged to refer to recent surveys $[4,9,10,16]$ that provide systematic classifications of various analysis and visualization techniques. In this section, we focus on the most relevant work that attempts to classify different integral curves based on a variety of similarity metrics.

Vector field topological analysis Vector field topology provides a streamline classification strategy based on the origin and destination of the individual streamlines. Since its introduction to the visualization community [8], vector field topology has received extensive attention. A large body of work has been introduced to identify different topological features, including fixed points $[17,26]$ and periodic orbits $[1,25,30]$. Recently, Chen et al. [2] studied the instability of trajectory-based vector field topology and, for the first time, proposed Morse decomposition for vector field topology computation, which leads to a more reliable interpretation of the resulting topological representation of vector fields. Szymczak et al. [24] introduced a new approach to converting the input vector field to a piecewise constant (PC) vector field and computing the Morse decomposition on triangulated manifold surface.

For the topological analysis of unsteady flows, Lagrangian Coherent structures (LCS), i.e., curves (2D) or surfaces (3D) in the domain across which the flux is negligible, were introduced to identify separation structures in unsteady flows. The computation of LCSs was first introduced by Haller [7] by computing the Finite Time Lyapunov Exponent (FTLE) of the flow, whose ridges indicate the LCS. Since its introduction, FTLE has been compared with the separatrices in the steady cases [19], and its computational performance has been improved substantially [5].

Streamline and pathline attributes Sadarjoen and Post introduced the winding angle concept for streamlines and utilized it to classify the streamlines within vortical regions [18]. Salzbrunn and Scheuermann introduced streamline predicates, which classify streamlines by interrogating them as they pass through certain user-specified features, e.g., vortices [21]. Later, this approach was extended to the classification of pathlines [20]. At the same time, Shi et al. [23] presented a data exploration system to study the characteristics of pathlines based on various attributes, including winding angle. Recently, a statistics-based method was proposed to help select the proper set of pathline attributes to improve the interactive flow analysis [15]. Our work differs from pathline predicate and pathline attribute approaches in that it utilizes the attributes of the individual pathlines to construct a smooth scalar field to classify the spatial locations where the pathlines are seeded. This enables us to study the flow structure as well as to classify the integral curves via the behavior of the derived scalar field. More recently, McLoughlin et al. [14] introduced the idea of a streamline signature based on a set of curve-based attributes including curvature and torsion. This streamline signature is used as a measure of the similarity between streamlines, and helps domain experts place and filter streamlines for the creation of an informative and uncluttered visualization of $3 \mathrm{D}$ flow.

\section{Vector Field Background and $\Phi$-Field}

Considering a spatio-temporal domain $\mathbb{D}=\mathbb{M} \times \mathbb{T}$ where $\mathbb{M} \subset \mathbb{R}^{d}$ is a $d$-manifold ( $d=2,3$ in our cases) and $\mathbb{T} \subset \mathbb{R}$, a vector field can be expressed as an ordinary differential equation (ODE) $\dot{\mathbf{x}}=V(\mathbf{x}, t)$ or a map $\varphi: \mathbb{R} \times \mathbb{M} \rightarrow \mathbb{R}^{d}$, satisfying $\varphi_{t_{0}}^{t_{0}}(\mathbf{x})=\mathbf{x}_{\mathbf{0}}$ and $\varphi_{t_{0}}^{t+s}(\mathbf{x})=\varphi_{s}^{t+s}\left(\varphi_{t_{0}}^{s}(\mathbf{x})\right)=\varphi_{t}^{t+s}\left(\varphi_{t_{0}}^{t}(\mathbf{x})\right)$.

There are a number of curves that describe different aspects of translational properties of vector fields, including streamlines, pathlines, streaklines and timelines. In this work, we focus only on streamlines and pathlines derived from the given vector fields. For a steady vector field $V(\mathbf{x})$, a streamline is a solution to the initial value problem of the ODE system confined to a given time $t_{0}: \mathbf{x}_{t_{0}}(t)=\mathbf{p}_{0}+\int_{t_{0}}^{t} V\left(\mathbf{x}(\eta) ; t_{0}\right) d \eta$. Based on the definition of streamlines, a number of features can be defined. A point $x_{0} \in \mathbb{M}$ is a fixed point (or singularity) if $\varphi\left(t_{0}, x\right)=x$ for all $t \in \mathbb{R}$. That is, $V\left(x_{0}\right)=0 . x$ is a periodic point if there exists $T>0$ such that $\varphi(T, x)=x$. The trajectory (or streamline) of a periodic point is called a periodic orbit. Fixed points, periodic orbits and their connectivity define the vector field topology [1]. For an unsteady vector field $V(\mathbf{x}, t)$, the trajectory of a particle starting at $\mathbf{x}_{0}$ and at time $t_{0}$ is called a pathline, denoted by $\mathbf{x}_{\mathbf{x}_{0}, t_{0}}(t)=\mathbf{x}_{0}+\int_{0}^{t} V\left(\mathbf{x}_{\mathbf{x}_{0}, t_{0}}(\tau), t_{0}+\tau\right) d \tau$.

\section{$\Phi$ Field and Its Gradient}

Various attributes can be extracted for the analysis and classification of integral curves [15]. Among these attributes, many can be obtained by integrating certain local flow properties measured along the integral curve, such as the acceleration, divergence, the arc-length and the winding angle. We adopt the Eulerian representation from texture-based methods and store the accumulated values at the sampled spatial positions. The value at each sample position is determined by the attribute value of the integral curve that is initiated at it. This Eulerian representation gives rise to a derived attribute field. Physically, the attribute computation and visualization framework is a way to look at the accumulated behavior of the individual particles along their trajectories. Therefore, it is helpful for revealing some long-term and global flow behaviors (like separation).

Considering an integral curve, $\mathscr{C}$, that starts from a given spatio-temporal point $\left(\mathbf{x}, t_{0}\right)$, the attribute field value at this point is computed as [32]:

$$
\mathscr{F}(\mathbf{x}, T)=\mathscr{F}\left(\left.\mathscr{C}(\mathbf{x})\right|_{t_{0}} ^{t_{0}+T}\right)
$$

where $\left.\mathscr{C}(\mathbf{x})\right|_{t_{0}} ^{t_{0}+T}$ denotes an integral curve, i.e., either a streamline or a pathline starting at time $t_{0}$ with an integral time window $\left[t_{0}, t_{0}+T\right] . \mathscr{F}(\cdot)$ indicates a specific attribute of interest of $\mathscr{C}$. It needs to be emphasized that the value of the attribute field assigned to a given spatio-temporal point $\left(\mathbf{x}, t_{0}\right)$ is associated with the motion of a particle that is seeded at that point $\mathbf{x}$ at time $t_{0}$. Therefore, in an unsteady flow, a different attribute field value can be obtained for a different $t_{0}$. Assume that an integral curve $\mathscr{C}$ is represented by $N$ integration points $P_{i}$ and $(N-1)$ line segments $\left(P_{i}, P_{i+1}\right)$. The $\Phi$ field and its gradient are computed as follows. 

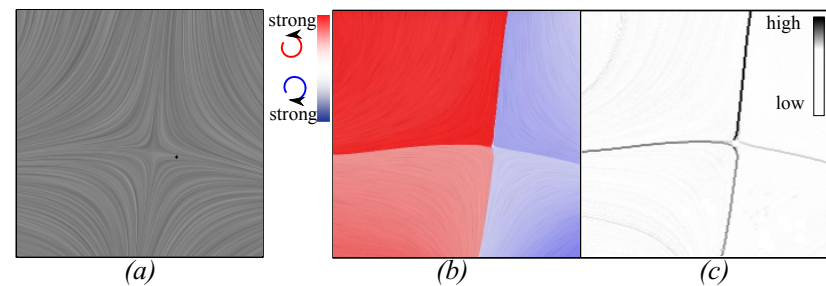

Figure 1: An example of $\Phi$ field (b) and its gradient magnitude field $|\nabla \Phi|$ (c). (a) shows the LIC of the flow.

$\Phi$ Field: The $\Phi$ field is determined from:

$$
\Phi_{\mathscr{C}}=\sum_{i=1}^{N-1} d \theta_{i}
$$

where for $2 \mathrm{D}$ vector fields $d \theta_{i}=\left(\angle\left(\overrightarrow{P_{i} P_{i+1}}, \vec{X}\right)-\right.$ $\left.\angle\left(\vec{P}_{i-1} P_{i}, \vec{X}\right)\right) \in(-\pi, \pi]$ represents the angle difference between two consecutive line segments on an integral curve. $\vec{X}$ is the $\mathrm{X}$ axis of the $\mathrm{XY}$ Cartesian space. $d \theta_{i}>0$ if the vector field at $P_{i}$ is rotating counter-clockwise with respect to the vector field at $P_{i-1}$, while $d \theta_{i}<0$ if the rotation is clockwise. For 3D vector fields, we use $d \theta_{i}=\operatorname{arcos}\left(\frac{\overrightarrow{P_{i} P_{i+1}} \bullet \overrightarrow{P_{i-1} P_{i}}}{\left|\overrightarrow{P_{i} P_{i+1}}\right|\left|\vec{P}_{i} P_{i+1}\right|}\right)$, i.e., the absolute difference of the direction of the two consecutive line segments on an integral curve. From a global perspective, the $\Phi$ field describes the total signed rotation for 2D flows and total absolute rotation for 3D flows along the trajectory. Figure 1(b) shows an example of the $\Phi$ field visualized using a blue-white-red color coding with blue color corresponding to the negative $\Phi$ values, red for positive, and white for zero values.

$|\nabla \Phi|$ Field: The gradient of the $\Phi$-field is defined as $\nabla \Phi=$ $\left(\frac{\partial \Phi}{\partial x}, \frac{\partial \Phi}{\partial y}, \frac{\partial \Phi}{\partial z}\right)$. It is well-known that $\nabla \Phi$ points in the direction where $\Phi$ increases the fastest, and its magnitude $|\nabla \Phi|$ indicates the rate of variation of $\Phi$ in this direction. Figure 1(c) shows a visualization of the gradient of the $\Phi$-field shown in Figure 1(a). A gray scale color coding is used with black corresponding to larger $|\nabla \Phi|$ values. The detailed computation of both the $\Phi$ and $|\nabla \Phi|$ fields will be discussed later.

In the following, we discuss a number of important properties of the $\Phi$ and $|\nabla \Phi|$ fields.

Property 1: Existence and Uniqueness of $\Phi$ value - One important property of the $\Phi$ field is that given any point $(\mathbf{x}, t) \in \mathbb{D}$ (except at fixed points in steady flows), there is exactly one $\Phi$ value returned by Eq.(2) given the specified parameters, i.e., the starting time $t_{0}$ and the time window $T$ for the integral curve computation. This is because in theory there exists exactly one integral curve passing through any given point at a given time except at fixed points. This property may seem trivial but it indicates that $\Phi$ field achieves complete spatial coverage, which enables us to generate a dense visualization of the flow.

Property 2: Discontinuity - This $\Phi$ field needs not be continuous everywhere in $\mathbb{D}$. For a steady vector field that contains fixed points, the integral curves (or streamlines) initiated at the fixed points reduce to points. Therefore, the obtained $\Phi$ field is not continuous at these locations. Also, the $\Phi$ field is not continuous across the integral curves that end or start from saddles, i.e., separatrices-a special class of streamlines, if the accumulation is performed infinitely over time. This is because an arbitrarily small perturbation in the direction other than the flow direction will lead to another integral curve with a length much different from the separatrix, making the $\Phi$ field accumulated using Eq.(1) discontinuous at separatrices. For an unsteady flow, sharp changes in the $\Phi$ field may still be observed at the ridges of transportation, i.e., the Lagrangian coherent structure (LCS). The reason is similar to the case of the separatrices. We refer to this sharp change as a discontinuity. This discontinuity can be captured by the $|\nabla \Phi|$ fields, where they correspond to places with large $|\nabla \Phi|$ values (e.g. the ridges with darker colors shown in Figures 1(c), 6(b) and 7(b)). Other discontinuities that are not related to the flow topological structure may also arise. For example, Figure 8(b) shows some cusp-like discontinuity structure in the $\Phi$ field caused by the sharp change of the direction of the pathlines. Details are provided in a later section.

Property 3: Inequality Property - To study this property, we assume the time window $T$ is either the whole time range for an unsteady flow or $\infty$ for a steady flow. By the definition of the $\Phi$ field (and other attribute fields), all the points at a given time $t_{0}$ that are correlated via the same integral curve get the same $\Phi$ value, while neighboring points that are not correlated by the same integral curve may have different values. In this case, one will expect the inequality $\left|\left\langle\nabla \Phi, V^{\perp}\right\rangle\right|>|\langle\nabla \Phi, V\rangle|$. This inequality depicts that the change of $\Phi$ along flow direction is smaller than along a direction perpendicular to the flow direction. This inequality is employed to evaluate the quality of the texture-based flow visualization techniques [13]. However, this may not be always satisfied due to the discrete sampling and the smoothing or blurry effect during the accumulation in practice. Therefore, we weaken the above inequality and introduce the following inequality for our study.

$$
|\nabla \Phi|>\left|\left\langle\nabla \Phi, \frac{V}{\|V\|}\right\rangle\right|
$$

In this case, the patterns in $|\nabla \Phi|$ is aligned with the flow direction. This property enables us to study the flow patterns based on the difference between the accumulated (or average) behaviors of neighboring integral curves, e.g., the extraction of the boundary of different flow regions.

Figure 2 illustrates how the above properties of the $\Phi$ and $|\nabla \Phi|$ fields may be used to highlight separation structure, vortices, and boundary transitions in the steady vector fields. In these examples, we sample the $\Phi$ and $|\nabla \Phi|$ fields along the specified line segments (shown in red). The $\Phi$ and $|\nabla \Phi|$ values distributed along these line segments are shown in the second and third columns of Figure 2, respectively. We can see that the $\Phi$ field exhibits sharp changes across these features. This is captured by the $|\nabla \Phi|$ field, as indicated by those spikes in the plots. More examples and discussion on the relation between the $\Phi$ and $|\nabla \Phi|$ fields and these well-known steady flow features will be given in Section 5 .

\section{Relating the $\Phi$ field to other attribute fields}

$\Phi$ vs. Curvature For this $\Phi$ field, Equation 2 is intrinsically related to the curvature of the integral curves. This is because the curvature, $\kappa(s)$, at any given point $\mathscr{C}(s)$ is $\kappa(s)= \pm\left|T^{\prime}(s)\right|$ ( $T$ is the tangent at $\mathscr{C}(s)$ ), and $\left|T^{\prime}\left(s_{0}\right)\right|=\left|\frac{T\left(s_{0}+d s\right)-T\left(s_{0}\right)}{d s}\right|=$ 


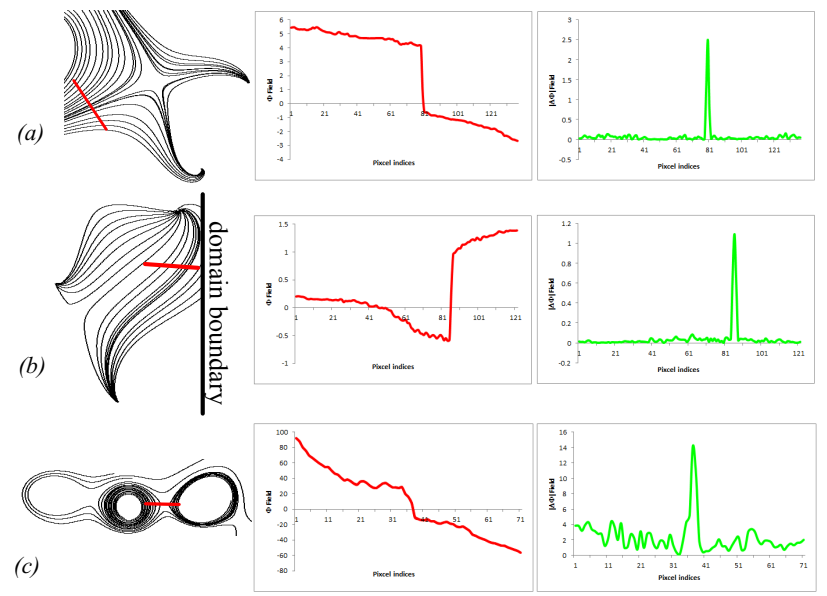

Figure 2: The illustration of the relation between the ridges in the $|\nabla \Phi|$ field and a number of well-known flow features, including the flow separation (a), boundary transition (b), and vortices (c). The left column shows the vector fields illustrated by streamlines, while the right column shows the plots of the $\Phi$ values (middle) of the streamlines intersecting with some seeding line segments (shown in red) and the corresponding $|\nabla \Phi|$ values (right). Note that the sharp changes in $\Phi$ field are captured by the $|\nabla \Phi|$ field.

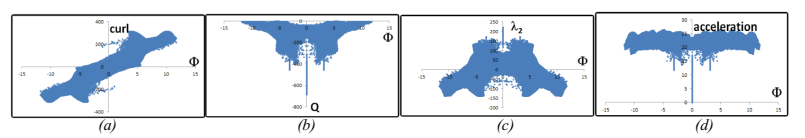

Figure 3: The scatter plot between the $\Phi$ field and curl (a), $Q$ (b) and $\lambda_{2}$ (c) and acceleration field (d), respectively, for the Double Gyre flow.

$\left|\frac{\tilde{V}\left(\mathscr{C}\left(s_{0}+d s\right)\right)-\tilde{V}\left(\mathscr{C}\left(s_{0}\right)\right)}{d s}\right| \approx\left|\frac{d \theta}{d s}\right|$ (if $d s \rightarrow 0$ ), where $\tilde{V}\left(\mathscr{C}\left(s_{0}\right)\right)$ is the normalized velocity vector defined at $\mathscr{C}\left(s_{0}\right)$, and $d \theta$ denotes the angle change between $\tilde{V}\left(\mathscr{C}\left(s_{0}+d s\right)\right)$ and $\tilde{V}\left(\mathscr{C}\left(s_{0}\right)\right)$. This equation holds because an integral curve in the flow field, such as a streamline or a pathline, is tangent to the vector field everywhere.

$\Phi$ vs. curl, $\lambda_{2}, \mathbf{Q}$ curl, $\lambda_{2}$ and $Q$ fields are three fields where the specific attributes in Equation 1 are the $\mathrm{curl}, \lambda_{2}$ and $Q$ measured at each sampled point, respectively [32]. $\lambda_{2}$ and $Q$ are two local criteria for the identification of vortices [3]. These four attributes are closely related, as they provide quantitative flow rotation information in different ways. Figure 3 (a-c) show the scatter plots with $\Phi$ as the $\mathrm{X}$ axis, and curl, $\lambda_{2}$ and $Q$ as the $\mathrm{Y}$ axis, respectively. All the plots exhibit certain symmetric patterns and tend to reveal clean patterns with little noise. This shows that $\Phi$ field encodes similar flow rotation information as those measured using other well-known metrics.

$\Phi$ vs. Acceleration Field $A$ Figure 3 (d) shows the scatter plot with $\Phi$ field as the $\mathrm{X}$ axis and acceleration field $A$ as the $\mathrm{Y}$ axis. Here, the $A$ field is obtained by integrating the acceleration magnitude along the individual pathlines. From this scatter plot, we see a clear pattern. In particular, when the absolute value of the $\Phi$ field is large, the $A$ field tend to be large as well. This is consistent with the knowledge that the acceleration, a result of the external force based on Newton Second Law, is the source of many important flow behaviors including rotation.

LCC based correlation In addition to the scatter plots, we quan-

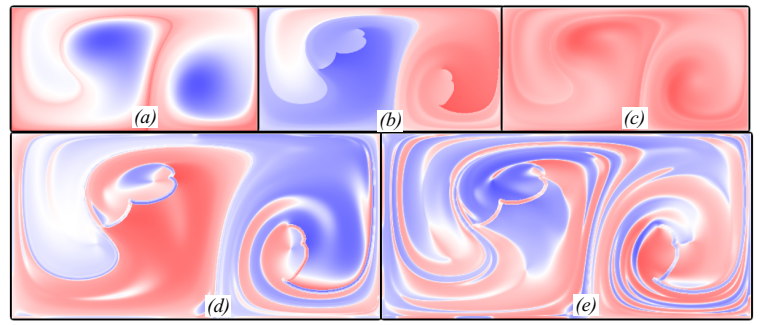

Figure 4: The correlation between the $\Phi$ field and other fields. (a)-(c) show the $\lambda_{2}$ field, $\Phi$ field and acceleration field of the Double Gyre flow, respectively. (d) is the correlation between the $\Phi$ field and $\lambda_{2}$ field. (e) is the correlation between the $\Phi$ field and acceleration field. Both are computed with local correlation coefficient.

tify the correlation between $\Phi$ field and other attribute fields based on the Local Correlation Coefficient (LCC) introduced by Sauber et al. [22]. For a local spatial position $\mathbf{x} \in \mathbb{M}$, we use a window function $G_{(\mathbf{x})}$. The local correlation coefficient between the $\Phi$ field and the acceleration field $A$ is defined by:

$$
\begin{aligned}
\operatorname{LCC}_{\Phi, A}(\mathbf{x}) & =\rho_{\Phi, A}(\mathbf{x})=\frac{\operatorname{cov}_{\Phi, A}(\mathbf{x})}{\sigma_{\Phi}(\mathbf{x}) \sigma_{A}(\mathbf{x})} \\
\operatorname{cov}_{\Phi, A}(\mathbf{x}) & =\int_{\mathbf{x} \in \mathbb{M}} G_{(\mathbf{x})}(\mathbf{x})(\Phi(\mathbf{x})-\bar{\Phi}(\mathbf{x}))(A(\mathbf{x})-\bar{A}(\mathbf{x})) d \mathbf{x} \\
\sigma_{A}(\mathbf{x}) & =\sqrt{\operatorname{cov}_{A, A}(\mathbf{x})} \\
\bar{A} & =\int_{\mathbf{x} \in \mathbb{M}} G_{(\mathbf{x})}(\mathbf{x}) A(\mathbf{x}) d \mathbf{x}
\end{aligned}
$$

$L C C_{\Phi, A}(\mathbf{p})$ returns a real number in the range $[-1,1]$. The closer to 0 , the weaker is the correlation between $\Phi$ and $A$. $\Phi$ and $A$ are positively correlated if $L C C_{\Phi, A}(\mathbf{p})>0$; otherwise, they are negatively correlated. We use a circular filter with radius $r$ as the window function $G_{(\mathbf{x})}$. The LCC coefficient between $\Phi$ field and other attribute fields can be computed similarly using Equation 4. Figure 4(d) shows the LCC correlation between the $\Phi$ field and the $\lambda_{2}$ field. Because both fields encode rotation information of the flow, they are highly correlated in the vortex areas (reflected by large regions with similar colors in Figure 4(d)). However, the $\Phi$ field also encodes the information of flow rotation orientations, which is not captured by the $\lambda_{2}$ field, therefore, the LCC correlation can be both positive (red) and negative (blue). Note that the discontinuity in LCC correlation is caused by the discontinuity in the $\Phi$ field. Figure 4(e) shows the LCC correlation between the $\Phi$ field and the acceleration field, which exhibits patterns that are mostly aligned with the flow direction due to the aforementioned Property 3 of the attribute fields. Again, except for the discontinuity caused by the $\Phi$ field, both fields have large correlation near vortex regions. However, the interleaving positive (red) and negative (blue) correlation regions outside the vortices will need in-depth investigation.

The above correlation studies indicate that $\Phi$ field may be an important attribute field that encodes different geometric and physical information of the flow for subsequent data exploration. 


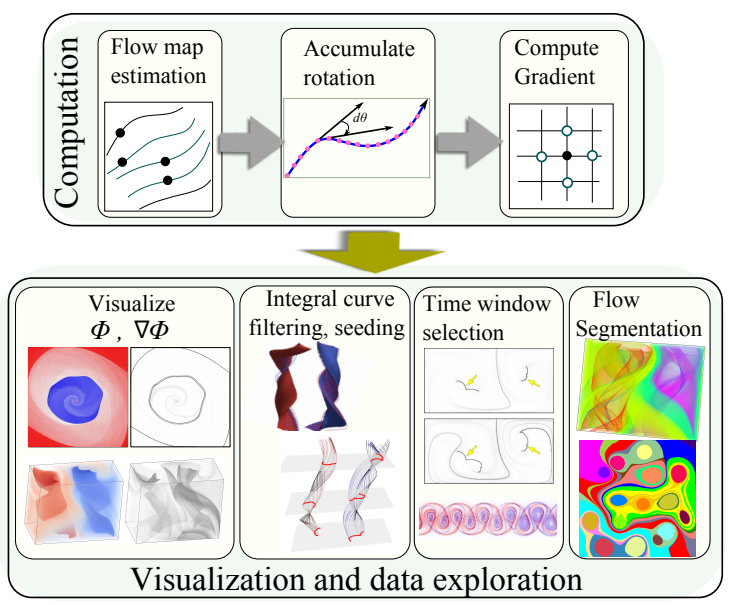

Figure 5: $\Phi$ field based analysis and visualization framework.

\section{A $\Phi$ Field-based Flow Exploration Frame- work}

Figure 5 shows the framework for the $\Phi$ field-based visualization and exploration strategy. This framework consists of two components: 1) computation of the $\Phi$ field and its gradient and 2) visualization and data exploration based on the $\Phi$ field and its gradient.

\section{Computing $\Phi$ and $|\nabla \Phi|$}

The computation of the $\Phi$ field starts with a flow map estimation. In order to capture the detailed flow information, we employ a regular sampling strategy. That is, we partition the domain into $N_{X} \times N_{Y} \times N_{Z}$ grid cells. In our experiments, $N_{X} \times N_{Y} \times N_{Z}$ matches the resolution of the image plane in $2 \mathrm{D}$ or the original resolution of the data in 3D unless stated otherwise. From each seed $\mathbf{x}$ (at the center of each cell), an integral curve is computed using a $4^{t h}$-order Runge-Kutta integrator. A linear interpolation scheme is applied in both space and time during integration.

We separate the termination conditions for streamline and pathline computations based on the definition of the $\Phi$ fields for steady and unsteady flows. For steady flows, given a starting point $\left(x_{0}, y_{0}\right)$, a streamline is computed in both forward and backward flow directions until it 1) reaches a boundary of the data domain; 2) reaches a singularity; 3 ) forms a closed loop; or 4) reaches the maximum number of integration time (or steps) $T$. Note that criterion 3) is needed because the unpredictably large $\Phi$ value near a center-type singularity may make the visualization of the $\Phi$-field challenging. For unsteady flows, given a starting point $\left(x_{0}, y_{0} ; t_{i}\right)$, a pathline is computed in both positive and negative time until it reaches a boundary of the data domain $\mathbb{D}$, e.g., the boundary of the physical domain or the boundary of the time range. In other words, we compute a complete pathline for each sample position.

After computing the integral curves starting from the sampled positions with the given integration time (or integration steps) $T$, we accumulate the flow rotation along the integral curve based on Equation 2. Since a regular sampling strategy is employed, $\nabla \Phi$ can be estimated by evaluating the central difference along each axis direction.

\section{$\Phi$ and $|\nabla \Phi|$ based Flow Exploration}

Integral curve filtering: We provide the user an interface to highlight the regions with a specified rotation behavior. Specifically, the user can adjust two thresholds, $\alpha$ and $\beta$, for the $\Phi$-field visualization, so that regions with $\alpha<\Phi<\beta$ will be colored white. Figure 8 (e) provides an example of this type of visualization. Because all points at a given time $t_{0}$ on the same integral curve have the same or similar $\Phi$ values, performing the above filtering is equivalent to eliminating integral curves whose $\Phi$ values are less than the threshold. However, this may still generate a set of integral curves that are too dense. To address this, we evenly subdivide the data ranges $\left[\Phi_{\min }, \alpha\right]$ and $\left[\beta, \Phi_{\max }\right]$ into $M$ intervals where $M$ is specified by the user. For each interval, an integral curve whose $\Phi$ value is closest to the median of the data range will be shown. With this interface, the user can easily highlight regions with strong positive and negative rotations that may indicate the vortices in the flow. Figure 9 (a) shows the vortex regions highlighted by the $\Phi$ field.

Integral curve seeding: We can utilize the inequality property and the discontinuity in the $\Phi$ fields to guide the generation of seeds for integral curves. For a $2 \mathrm{D}$ flow, we place seeds at the points where the $|\nabla \Phi|$ value is larger than a user-specified threshold, e.g., $0.8|\nabla \Phi|_{\max }$, where $|\nabla \Phi|_{\max }$ is the maximal gradient magnitude in the domain. Integral curves are then computed from these seeds. We start randomly from one seed, and filter out those seeds that are too close and proceed to the remaining seeds. We repeat this process until no more seeds are available. Figure 8(d) shows an example of the seeded pathlines in an unsteady flow.

Stream surface placement: For a 3D steady flow, we generate the candidate seeding curves for stream surface placement as follows. We first identify all the voxels with $|\nabla \Phi|$ values larger than a user-specified threshold. We then generate a seeding curve by computing a streamline segment from the center of a selected voxel in both forward and backward directions of a derived 3D vector field $V^{\perp}$, where $\left\langle V^{\perp}, V\right\rangle=0$. The integration is terminated if the length of the streamline is larger than a threshold (i.e., 20 steps in our experiments). This results in a set of candidate seeding curves. The user can then place stream surface(s) by selecting one or more seeding curves. Figure 12 provides an example of a seeded stream surface for the Lorenz system.

Flow segmentation: Based on Property 1, each spatio-temporal position $\left(\mathbf{x}, t_{0}\right)$ has a unique $\Phi$ value, so we can first classify the sampled spatial positions according to their $\Phi$ values. Then, the connected components of this classification are extracted to provide an initial segmentation. This initial segmentation may contain some smaller segments due to the numerical error in the attribute field computation. We then perform dilation operations to remove those small segments. Figure 11 provides an example of this segmentation. A detailed description of this segmentation algorithm is described in [33].

\section{Results and Applications}

We have applied $\Phi$ field-based flow exploration to a number of synthetic and real-world $2 \mathrm{D}$ and $3 \mathrm{D}$ vector fields. In this section, we will first discuss how visualizing the $\Phi$ field and its 
gradient field can be used to highlight certain well-known flow features. Then, we will show how the $\Phi$ field and its gradient can be used to support the aforementioned flow exploration tasks. The computation cost of our implementation is determined largely by the sampling resolution and the integration time (or the number of steps) $T$ employed. The computation time for each flow data set we employed in this work is listed in table 1. The computation is carried out on a PC with Intel(R) Xeon(R) $3.6 \mathrm{GHz}$ quad-core processor and 16GB RAM.

Table 1: The computation time of derived data sets in this work.

\begin{tabular}{llll} 
Data set & Resolution & $\begin{array}{l}\text { Number } \\
\text { of Steps }\end{array}$ & $\begin{array}{l}\text { Computation } \\
\text { Time }\end{array}$ \\
\hline synthetic flows & $512 \times 512$ & 2000 & 30 seconds \\
HCCI slice & $640 \times 640$ & 2000 & 70 seconds \\
Cylinder flow & $400 \times 50 \times 1001$ & 2000 & 3 hours \\
Double Gyre flow & $512 \times 256 \times 200$ & 200 & 50 minutes \\
Bernard flow & $128 \times 32 \times 64$ & 10000 & 20 minutes \\
Lorenz flow & $64 \times 64 \times 64$ & 15000 & 15 minutes
\end{tabular}

\section{Visualization of $\Phi$ and $|\nabla \Phi|$ Field}

The visualization of the $\Phi$ field is straight forward. For 2D flows, the $\Phi$ field can be shown using color plots or volume rendering with a blue-white-red color coding, where blue represents negative rotation and red represents positive rotation (Figure 6(a), Figure 7(a), Figure 8(a)). For 3D flows, since the $\Phi$ field is always positive, we can visualize it using volume rendering with a standard rainbow color coding where blue maps small attribute values and red indicates large attribute values (Figure 9(a), Figure 12 (b)). The $|\nabla \Phi|$ field is always visualized with a gray scale color coding (Figure 6(b), Figure 7(b), Figure 8(b), Figure 9(b), Figure 12 (c)).

Figure 6 shows two slices of cross sections from a diesel engine simulation [1]. Columns (a) and (b) provide the visualization of the $\Phi$ and $|\nabla \Phi|$ fields, respectively, while (c) shows the corresponding flow topology. From the comparisons, we see that the $|\nabla \Phi|$ field reveals flow structures similar to those obtained from a topological analysis. This is because the streamlines on different sides of a separatrix usually have different rotational behaviors, thus, leading to large variation in the $\Phi$ field that can be captured by $\nabla \Phi$ (Figure 2(a)). However, we emphasize that the ridges of the $|\nabla \Phi|$ field are features of the $\Phi$ field, which need not correspond to the original flow features. In practice, due to the varying density of integral curves convergence (divergence) of the flow, not all the points along the same integral curve have the same attribute value.

Figure 7 shows the volume rendering (top) and a number of 2D slices (bottom) of the $\Phi$ and $|\nabla \Phi|$ fields for the flow past a cylinder [27] using ParaView. This data set consists of 1001 time steps. To compute the $\Phi$ field, we uniformly sample $400 \times 50$ particles at each time step, and integrate the pathlines in both forward and backward time directions until they reach the domain boundary (i.e., $T=8$ ). For each pathline, the rotation integration starts from the position that the particle first enters the domain and along the positive time direction until it exits the domain. The measurement of each local rotation is performed by projecting the pathline onto the 2D plane where the vector field is defined. The computation of this $\Phi$ field took about 3 hours, and output file storing the pathlines is of size $184 \mathrm{MB}$. From the result, we can see that

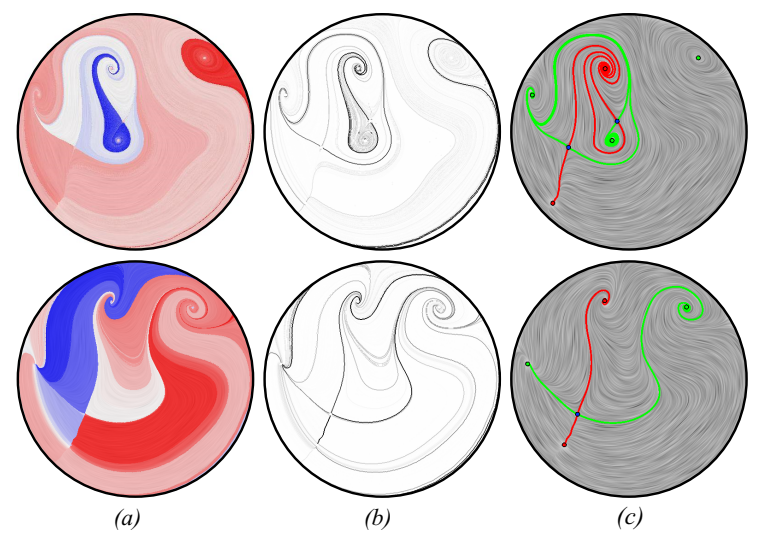

Figure 6: The analysis and visualization from the cross sections of a diesel engine simulation [11]. (a) $\Phi$ fields; (b) $|\nabla \Phi|$ field; (c) conventional topology.

(a)
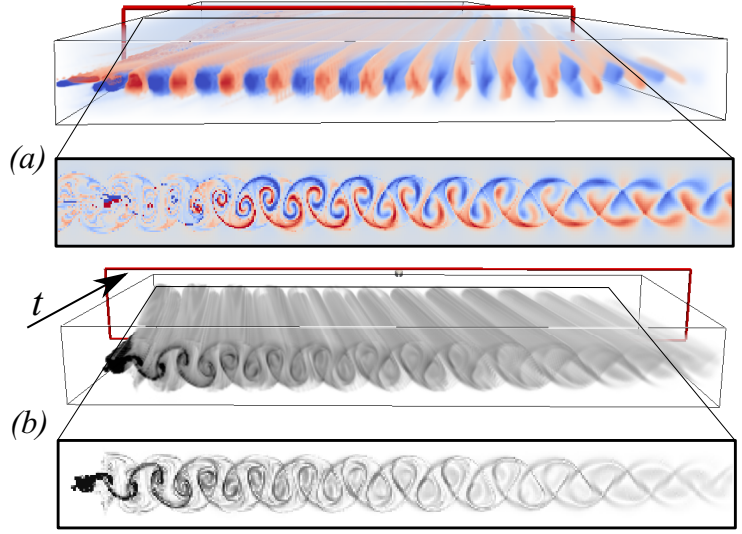

(c)

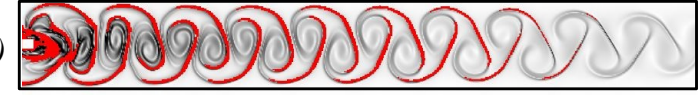

(d)

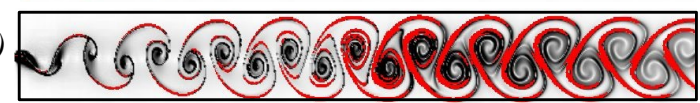

Figure 7: The visualization of the $\Phi$ (a) and $|\nabla \Phi|$ (b) fields of the cylinder flow data [27]. The time direction increases away from the view. The 2D slices correspond to time step $t_{0}=200$ are shown. (c) and (d) show the comparison of LCS and $|\nabla \Phi|$ filed computed based on forward pathlines (c) and backward pathlines (d) at $t_{0}=0$, respectively. The LCS is highlighted in red on top of the $|\nabla \Phi|$ visualization.

the $\Phi$ field (Figure 7(a)) reveals the regions with strong rotational behavior. The blue-white-red color coding effectively conveys the orientation of the rotation. The $|\nabla \Phi|$ field (Figure 7(b)) highlights the structure of the discontinuity in the $\Phi$ field, which assembles the combined forward and backward FTLE ridges, as shown in (c) and (d). The red pixels represent the LCS whose FTLE values are larger than $0.65 f_{\max }\left(f_{\max }\right.$ is the maximum FTLE value).

Figure 8 shows the results for the Double Gyre flow. In particular, Figure 8(a) shows the volume rendering of the $\Phi$ field, from which one can easily observe the strong rotational behavior of the pathlines that is induced by the two vortices. This can be better revealed by a set of pathlines selected via filtering the $\Phi$ field (Figure 8(e)). Figure 8 (b) shows the $|\nabla \Phi|$ field in the spatio-temporal domain. Two cross sections of this gradient field at $t=0$ and 20 are shown in the bottom two images of (b), re- 


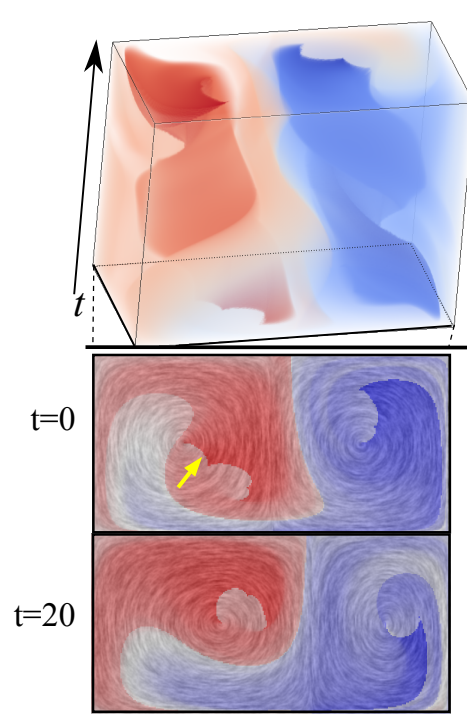

(a)
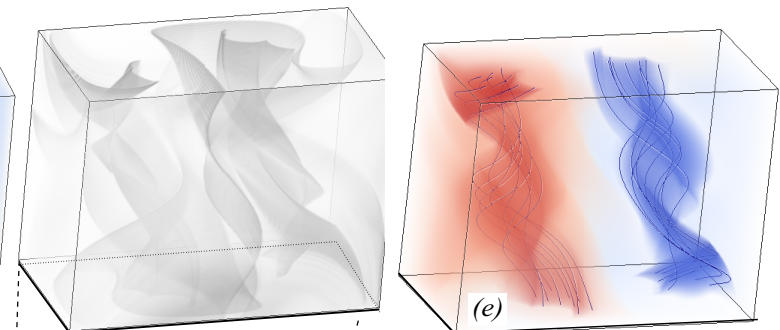

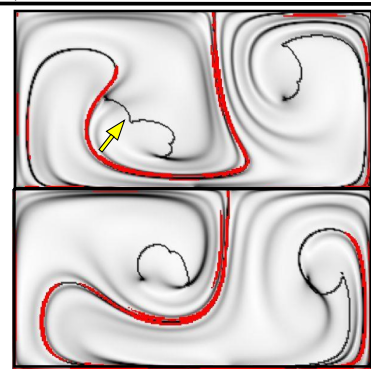

(b)

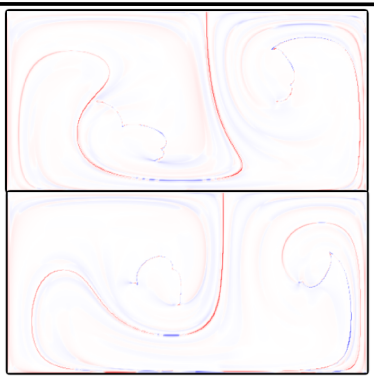

(c)
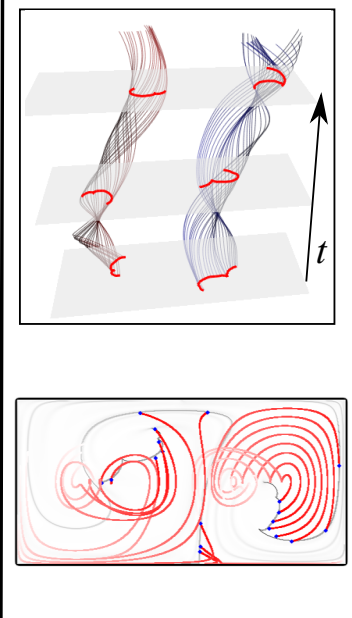

(d)

Figure 8: The result of the double gyre flow with $T=10$. (a-c) show the forward $\Phi$ field, the comparison between LCS and $|\nabla \Phi|$ ridges, and LCC based correlation between FTLE and $|\nabla \Phi|$ at time steps 0 and 20, respectively. For FTLE, a rainbow color coding is used where red indicates larger FTLE values and blue smaller. For the comparison of LCS and $|\nabla \Phi|$ ridges (b), the extracted FTLE ridge points are highlighted in red on top of the $|\nabla \Phi|$ visualization that utilizes a gray scale color map. In addition to the LCS structure, the $\Phi$ and $|\nabla \Phi|$ fields also capture the cusp-like seeding curves indicated by the yellow arrows. (d) shows the pathlines seeded at the cusp-like ridges of the $|\nabla \Phi|$ field. Note that the intersections of these pathlines with the (horizontal) time planes form some coherent structures rotating over time. (e) shows a set of selected pathlines by filtering the $\Phi$ field.

spectively, which are overlapped with the LCS ridges (i.e., the red pixels). From this visual comparison, we see that the $|\nabla \Phi|$ field captures behavior very similar to the FTLE field. It is worth noting that the proposed accumulation process may indicate the presence of a dynamic event (e.g., strong rotation) that occurs at a different location in the field and at a later time through the value assigned to the seeding location as long as they are correlated by the same pathline. In contrast, the FTLE field does not employ an accumulated value but instead represents the rate of divergence (convergence) of particles released from a region. The LCS, i.e., the ridges of the FTLE field, does accurately reflect the spatial location of the divergence. Nonetheless, our method does provide a classification for pathlines based on the similarity of their rotational behavior.

By close inspection, one also observes additional features in the $|\nabla \Phi|$ field that do not correspond to an LCS, that is, those cusp-like curves near the two vortices as highlighted by the arrow in Figure 8(b). They are essentially the cusp-like seeding curve discussed in [28]. This cusp-like behavior in pathlines is caused by the abrupt change in the pathline direction (i.e., almost angle of $\pi$ between the previous and current directions), which is in turn caused by the intersection of the pathlines with the paths of singularities. Such discontinuous behavior on the pathlines is captured by our $\Phi$ field with no additional cost.

Figure 9 (a) and (b) show the volume renderings of the $\Phi$ and $|\nabla \Phi|$ fields for the Benard data [29], whose domain is $[-16,16] \times[-4,4] \times[-8,8]$. Compared to the standard method for vortex identification, such as the $Q$ criterion [3] shown in Figure 9(d), our $\Phi$ and $|\nabla \Phi|$ field based visualizations reveal the eight vortices of this flow system clearly. In addition, the local attribute cannot reveal the global flow behaviors, that is, the full trajectories of the particles that enter the vortex regions. This global
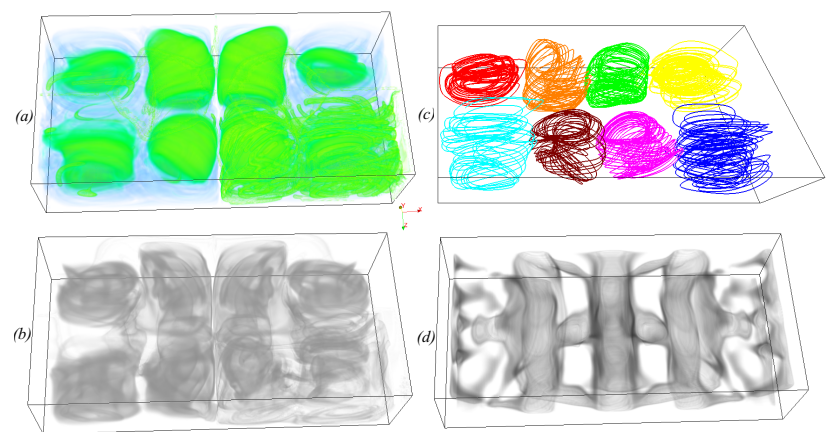

Figure 9: The volume rendering of the $\Phi$ field (a) and $|\nabla \Phi|$ field (b) of the Bernard flow. (c) shows several filtered streamlines based on the $\Phi$ field. (d) shows the local attribute and $Q$.
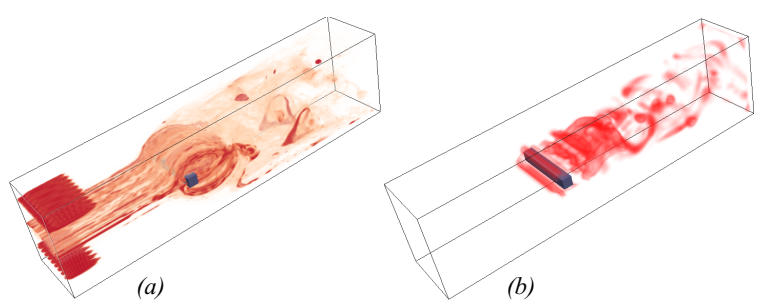

Figure 10: (a) The visualization of the $\Phi$ field of a 3D steady flow behind cylinder [27]. (b) Volume rendering of $Q$ for the same data set. A whitered color coding is applied.

information is captured by the $\Phi$ field. As shown in Figure 10(b), the visualization of $Q$ highlights the local vortices, while the $\Phi$ field in Figure 10(a) also reveals all particles which are eventually traced into the vortices regions in either forward or backward flow direction. Nonetheless, we note that the $\Phi$ field need not highlight the vortex regions identified by traditional methods. Rather, our 


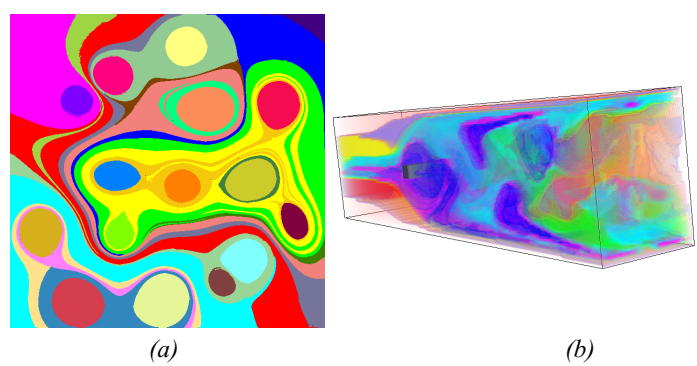

Figure 11: The $\Phi$ field based segmentation of a synthetic steady flow (a) and a 3D steady flow behind cylinder (b). The number of initial classifications is 10 and 5 , respectively.

aim is to utilize $\Phi$ field to help choose seeding particles that may yield vortex structure.

\section{Applications of $\Phi$ and $|\nabla \Phi|$ Field}

The above discussion on the similarity of certain features observed from the visualization of $\Phi$ and $|\nabla \Phi|$ fields to a number of well-known flow features is not meant to promote the $\Phi$ and $|\nabla \Phi|$ fields for explicit flow feature detection, but rather indicates that the derived $\Phi$ and $|\nabla \Phi|$ fields encode useful information that can be applied to a number of flow exploration tasks.

Integral curve filtering: In order to observe the strong rotation behaviors of a given flow, we can implement a simple filtering mechanism based on the $\Phi$ field, i.e., retaining the integral curves falling in regions with large absolute values of the $\Phi$ field. Figure 8(e) shows a set of pathlines obtained by filtering the $\Phi$ field of a Double Gyre flow, which have strong rotational behavior that is induced by the two vortices. The value range of $\Phi$ field for this filtering is $\left[\Phi_{\min }, 0.98 \Phi_{\min }+0.02 \Phi_{\max }\right]$ and $\left[0.02 \Phi_{\min }+0.98 \Phi_{\max }, \Phi_{\max }\right]$. Figure $9(\mathrm{c})$ shows a number of streamlines of the Bernard flow filtered by the $\Phi$ field. By keeping the streamlines with $\Phi$ values larger than $0.95 \Phi_{\max }$, we can select streamlines to reveal the strong rotation behavior in these regions, i.e., the eight vortices.

Integral Curve/Surface Seeding: Figure 8 (d) provides a pathline placement for the double gyre flow. The seeds of the pathlines are placed on the cusp-like seeding curves indicated by the yellow arrow in Figure 8 (b). Figure 12 (a) shows a seeded stream surface for the Lorenz system. The parameters are $\sigma=10, \rho=28$ and $\beta=8 / 3$, respectively. The domain of the Lorenz system is $[-30,30] \times[-30,30] \times[-10,50]$. The seeding curve (shown in red) is selected by the user from a number of candidate seeding curves (shown in green) generated based on the derived $|\nabla \Phi|$ field (c). Figure 12 (b) visualizes the corresponding $\Phi$ field.

Flow Segmentation: Figure 11(a) shows the segmentation of a synthetic flow based on the distribution of the $\Phi$ values. Ten segments are generated with the initial $m$ as 5 . From this segmentation, we can observe a few larger vortex systems that enclose a number of individual vortices, e.g., the vortex system enclosing a number of individual vortices. Visualizations like this may be used to reveal the hierarchy of vortices, leading to a level-ofdetail interpretation. Figure 11(b) shows the segmentation of a 3D steady flow behind cylinder based on the $\Phi$ field. This 3D steady flow is taken an instant from a simulation of a $3 \mathrm{D}$ unsteady flow

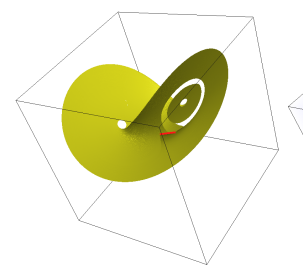

(a)

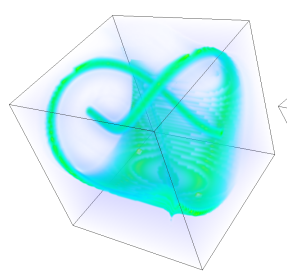

(b)

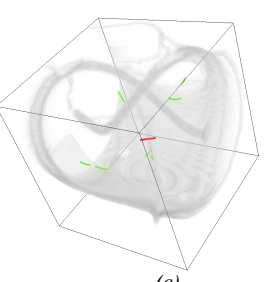

(c)
Figure 12: A stream surface placement of Lorenz flow based on $|\nabla \Phi|$ field (a). (b) and (c) are the visualization of $\Phi$ and $|\nabla \Phi|$ field, respectively. The seeding curve of the stream surface is highlighted with red color.

behind cylinder [27] at time step 102. Eight segments are generated with the initial $m=5$. With global information encoded in the $\Phi$ field, the spatial points which are not in the vortex regions but would be traced into the vortex regions are grouped together with the points in the vortex regions.

\section{Conclusion}

In this paper, we investigated the $\Phi$ field, a scalar field that is derived from the input vector field by integrating the rotation of the integral curves. We discussed a number of important properties of this field and its gradient, and showed how to use them to assist the visualization and exploration of 2D steady and unsteady flows and 3D steady flows. We described a framework for the computation of the $\Phi$ field and its gradient which can be applied to both streamlines and pathlines. We discussed the similarity and dissimilarity of the features observed in the $\Phi$ and $|\nabla \Phi|$ fields with those well-known features in the flow, and demonstrated how to utilize the encoded information in these derived fields to achieve integral curve selection, integral curve/surface seeding, and flow segmentation, via a number of synthetic and real-world flow data sets.

Despite its simplicity in computation and flexibility in data exploration, the $\Phi$ field computation has a number of limitations. First, it relies heavily on numerical integration. Therefore, numerical errors may compromise the results, especially in areas with large flow curvature. Second, it is possible that negative rotation may cancel the positive in some symmetric flow where the integral curves oscillate around an axis. Finally, the features of the $\Phi$ and $|\nabla \Phi|$ fields need not be the features of the flows as discussed earlier. Therefore, we consider the $\Phi$ field based analysis and visualization framework a valuable complement to the existing analysis and visualization techniques for vector fields. In the future, we aim at performing an in-depth investigation of the behaviors of these derived attribute fields and address the aforementioned limitations.

\section{Acknowledgments}

We thank Jackie Chen, Mathew Maltude, Tino Weinkauf for the data. This research was in part supported by NSF IIS-1352722 and IIS-1065107.

\section{Author Biography}

Lei Zhang received his M.S. degree in computer science and his B.S. degree in Administration of Industry from Xidian University, China, in 2009 and 2005, respectively. He is currently a PhD student at the Department of Computer Science at the University of Houston. His research interests are in the areas of flow 
visualization and data analytics.

Robert S. Laramee received a bachelors degree in physics, cum laude, from the University of Massachusetts, Amherst (Zoo Mass) and a masters degree in computer science from the University of New Hampshire, Durham. He was awarded a PhD from the Vienna University of Technology, Austria in 2005. He is an Associate Professor at the Swansea University, Wales in the Department of Computer Science. His research interests are in the areas of scientific visualization, information visualization, and visual analytics.

David Thompson holds the rank of professor in the Department of Aerospace Engineering at Mississippi State University. His previous related experience includes a faculty appointment at the University of Texas at Arlington. His research interests include computational fluid dynamics and visualization. He is the inaugural holder of the Airbus Helicopters, Inc. Professorship in Aerospace Engineering and was selected as a Royal Academy of Engineering Distinguished Visiting Fellow for 2014-15.

Adrian Sescu is currently an Assistant Professor of Aerospace Engineering at Mississippi State University. He received his Ph.D. in mechanical engineering from the University of Toledo, $\mathrm{OH}$, and his B.S. and M.S. in aerospace engineering from Polytechnic University of Bucharest. Before joining the ASE Department, Adrian was a postdoctoral fellow at Johns Hopkins University, MD. His research areas include Turbulence in fluids, Computational Aeroacoustics, and Transition in boundary layers.

Guoning Chen is an Assistant Professor at the Department of Computer Science at the University of Houston. He earned his Ph.D. degree in Computer Science from Oregon State University in 2009. His research interests include scientific data analysis and visualization, geometric modeling, geometry processing, and physically-based simulation. He is a member of IEEE and ACM.

\section{References}

[1] G. Chen, K. Mischaikow, R. S. Laramee, P. Pilarczyk, and E. Zhang. Vector field editing and periodic orbit extraction using Morse decomposition. IEEE Transactions on Visualization and Computer Graphics, 13(4):769-785, Jul./Aug. 2007.

[2] G. Chen, K. Mischaikow, R. S. Laramee, and E. Zhang. Efficient Morse decompositions of vector fields. IEEE Transactions on Visualization and Computer Graphics, 14(4):848-862, Jul./Aug. 2008.

[3] R. Cucitore, M. Quadrio, and A. Baron. On the effectiveness and limitations of local criteria for the identification of a vortex. European Journal of Mechanics-B/Fluids, 18(2):261-282, 1999.

[4] M. Edmunds, R. S. Laramee, G. Chen, N. Max, E. Zhang, and C. Ware. Surface-based flow visualization. Computers \& Graphics, 36(8):974-990, 2012.

[5] C. Garth, A. Wiebel, X. Tricoche, K. I. Joy, and G. Scheuermann. Lagrangian visualization of flow-embedded surface structures. Computer Graphics Forum, 27(3):1007-1014, 2008.

[6] H. Guo, F. Hong, Q. Shu, J. Zhang, J. Huang, and X. Yuan. Scalable lagrangian-based attribute space projection for multivariate unsteady flow data. In Pacific Visualization Symposium (PacificVis), 2014 IEEE, pages 33-40. IEEE, 2014.
[7] G. Haller. Lagrangian coherent structures and the rate of strain in two-dimensional turbulence. Phys. Fluids A, 13:3365-3385, 2001.

[8] J. L. Helman and L. Hesselink. Representation and display of vector field topology in fluid flow data sets. IEEE Computer, 22(8):27-36, August 1989.

[9] M. Jiang, R. Machiraju, and D. Thompson. Detection and visualization of vortices. In The Visualization Handbook, pages 295-309. Academic Press, 2005.

[10] R. Laramee, H. Hauser, L. Zhao, and F. H. Post. Topology based flow visualization: the state of the art. In Topology-Based Methods in Visualization (Proceedings of Topo-in-Vis 2005), Mathematics and Visualization, pages 1-19. Springer, 2007.

[11] R. S. Laramee, D. Weiskopf, J. Schneider, and H. Hauser. Investigating swirl and tumble flow with a comparison of visualization techniques. In Proceedings of IEEE Visualization '04, pages 51-58, 2004.

[12] K. Lu, A. Chaudhuri, T.-Y. Lee, H. W. Shen, and P. C. Wong. Exploring vector fields with distribution-based streamline analysis. In Proceeding of PacificVis '13: IEEE Pacific Visualization Symposium, Sydney, Australia, march 2013.

[13] V. Matvienko and J. Kruger. A metric for the evaluation of dense vector field visualizations. Visualization and Computer Graphics, IEEE Transactions on, 19(7):1122-1132, 2013.

[14] T. McLoughlin, M. W. Jones, R. S. Laramee, R. Malki, I. Masters, and C. D. Hansen. Similarity measures for enhancing interactive streamline seeding. IEEE Transactions on Visualization and Computer Graphics, 19(8):1342-1353, 2013.

[15] A. Pobitzer, A. Lez, K. Matkovic, and H. Hauser. A statistics-based dimension reduction of the space of path line attributes for interactive visual flow analysis. In PacificVis, pages 113-120, 2012.

[16] A. Pobitzer, R. Peikert, R. Fuchs, B. Schindler, A. Kuhn, H. Theisel, K. Matkovic, and H. Hauser. The state of the art in topologybased visualization of unsteady flow. Computer Graphics Forum, 30(6):1789-1811, September 2011.

[17] K. Polthier and E. Preuß. Identifying vector fields singularities using a discrete hodge decomposition. In Mathematical Visualization III, pages 112-134. Ed: H.C. Hege, K. Polthier, 2003.

[18] I. Sadarjoen and F. Post. Geometric methods for vortex extraction. In Proc. EG/IEEE Visualization Symposium, 1999.

[19] F. Sadlo and R. Peikert. Efficient visualization of lagrangian coherent structures by filtered amr ridge extraction. IEEE Transactions on Visualization and Computer Graphics, 13(6):1456-1463, 2007.

[20] T. Salzbrunn, C. Garth, G. Scheuermann, and J. Meyer. Pathline predicates and unsteady flow structures. The Visual Computer, 24(12):1039-1051, 2008.

[21] T. Salzbrunn and G. Scheuermann. Streamline predicates. IEEE Transactions on Visualization and Computer Graphics, 12(6):16011612, 2006.

[22] N. Sauber, H. Theisel, and H.-P. Seidel. Multifield-graphs: An approach to visualizing correlations in multifield scalar data. Visualization and Computer Graphics, IEEE Transactions on, 12(5):917924, 2006.

[23] K. Shi, H. Theisel, H. Hauser, T. Weinkauf, K. Matkovic, H.-C. Hege, and H.-P. Seidel. Path line attributes - an information visualization approach to analyzing the dynamic behavior of 3D timedependent flow fields. In H.-C. Hege, K. Polthier, and G. Scheuermann, editors, Topology-Based Methods in Visualization II, Mathematics and Visualization, pages 75-88, Grimma, Germany, 2009. Springer. 
[24] A. Szymczak and E. Zhang. Robust morse decompositions of piecewise constant vector fields. Visualization and Computer Graphics, IEEE Transactions on, 18(6):938-951, 2012.

[25] H. Theisel, T. Weinkauf, and H.-P. Seidel. Grid-independent detection of closed stream lines in 2D vector fields. In Proceedings of the Conference on Vision, Modeling and Visualization 2004 (VMV 04), pages 421-428, Nov. 2004.

[26] X. Tricoche, G. Scheuermann, and H. Hagen. Continuous topology simplification of planar vector fields. In Proceedings of IEEE Visualization 2001, pages 159-166, 2001.

[27] T. Weinkauf and H. Theisel. Streak lines as tangent curves of a derived vector field. IEEE Transactions on Visualization and Computer Graphics (Proceedings Visualization 2010), 16(6):1225-1234, November - December 2010.

[28] T. Weinkauf, H. Theisel, and O. Sorkine. Cusps of characteristic curves and intersection-aware visualization of path and streak lines. In R. Peikert, H. Hauser, H. Carr, and R. Fuchs, editors, Topological Methods in Data Analysis and Visualization II, Mathematics and Visualization, pages 161-176. Springer, 2012.

[29] D. Weiskopf, T. Schafhitzel, and T. Ertl. Texture-based visualization of unsteady $3 \mathrm{~d}$ flow by real-time advection and volumetric illumination. Visualization and Computer Graphics, IEEE Transactions on, 13(3):569-582, 2007.

[30] T. Wischgoll and G. Scheuermann. Detection and visualization of closed streamlines in planar fields. IEEE Transactions on Visualization and Computer Graphics, 7(2):165-172, 2001.

[31] H. Yu, C. Wang, C.-K. Shene, and J. H. Chen. Hierarchical streamline bundles. IEEE Transactions on Visualization and Computer Graphics, 18(8):1353-1367, Aug. 2012.

[32] L. Zhang, R. S. Laramee, D. Thompson, A. Sescu, and G. Chen. Compute and Visualize Discontinuity Among Neighboring Integral Curves of 2D Vector Fields. In Proceedings of TopoInVis, Germany, 2015.

[33] L. Zhang, R. S. Laramee, D. Thompson, A. Sescu, and G. Chen. Vector Field Segmentation Based on Integral Curve Attributes. In Proceedings of ChinaVis, Tianjing, China, 2015. 